INFLAMMATORY BOWEL DISEASE

\title{
Isolation, maturational level, and functional capacity of human colon lamina propria plasma cells
}

\author{
F Medina, C Segundo, A Campos-Caro, I Salcedo, A García-Poley, J A Brieva
}

Gut 2003;52:383-389

See end of article for authors' affiliations

Correspondence to: J A Brieva, Servicio de Inmunología, Hospital Universitario Puerta del Mar, Avenida Ana de Viya 21, 11009 Cádiz, Spain; jabrieva@

hpm.sas.junta-andalucia.es

Accepted for publication 11 September 2002

\begin{abstract}
Background and aims: Large numbers of plasma cells (PC) localise in the intestinal lamina propria (LP) where they play a critical role in the defence against pathogens. This study analyses the level of maturation reached by normal human colon LPPC in comparison with that of bone marrow (BM) PC.

Methods: A technique was designed to purify LPPC by combining collagenase digestion of the mucosal layer and immunomagnetic selection of $\mathrm{CD} 54^{+} \mathrm{LP}$ cells. It provided highly purified PC, as demonstrated by morphology, CD38 $8^{\text {h }}$ phenotype, and cytoplasmic lgA staining criteria. This procedure allowed comparison of in vitro functional capacities and a broad phenotypic analysis of BMPC and LPPC.

Results: LPPC and BMPC exhibited identical expression of differentiation markers $\left(C D 19^{-/+}, C^{-} 20^{-}\right.$, HLA-DR ${ }^{\text {low/- }}$, VS38 $\left.\mathrm{c}^{\text {high }}\right)$, survival molecules $\left(C D 95^{\text {low/- }}, \mathrm{Bcl}-2^{+}\right)$, and B cell transcription factor profile, as well as similar in vitro lg secreting kinetics (14 days) and lack of susceptibility to apoptosis by CD95 ligation. In contrast, they markedly differed in adhesion molecule expression, as LPPC showed higher levels of CD44 and CD2 1 and were $\alpha 4 \beta 7^{+}$whereas BMPC lacked this integrin and expressed higher levels of CD49d and CD31.

Conclusion: These data indicate that PC at effector sites of the humoral response (BM and LP) show similar high differentiation, survival, and functional features but display a distinctive pattern of adhesion molecules, probably related to their respective homing locations.
\end{abstract}

$P$ lasma cells (PC) are considered the final stage of the B lymphocyte differentiation process and as such show features that reveal their full specialisation in the synthesis and secretion of antibodies (Ab). Therefore, $\mathrm{PC}$ are ultimately responsible for the formation of $\mathrm{Ab}$ in response to antigens and for adequate maintenance of the Ab level of the organism. In animal models of systemic humoral immune responses, specific PC initially appear in antigen activated foci and germinal centres of inductive lymphoid organs (lymph nodes, spleen). ${ }^{1-3}$ Subsequently, some of these cells are thought to migrate into the bone marrow (BM), the final deposit and effector organ for PC elicited in systemic responses. ${ }^{45}$ Human $\mathrm{BM}$ also becomes the final reservoir of PC formed in distant inductive territories following systemic antigen stimulation and, accordingly, human BMPC generate the majority of serum Ig formation. ${ }^{6}$ Although PC present in different organs show a similar morphology and Ig secreting role, evidence increasingly demonstrates the existence of relevant differences within the PC compartment. In rodent models, BMPC show a prolonged lifespan (from several weeks to several months) while the majority of PC present in inductive organs die in 2-3 days, ${ }^{7-9}$ apparently by apoptosis. ${ }^{10}$ In addition, PC exhibiting IgVH gene somatic mutations that confer higher affinity for the antigen are progressively enriched in the BMPC pool during the course of the response. ${ }^{11}{ }^{12}$ In humans, PC occurring in peripheral lymphoid organs such as lymph nodes, blood, and tonsils show short term kinetics of Ig secretion in vitro $^{13-16}$ and exhibit a propensity to undergo apoptosis either spontaneously or induced by CD95 cross linking ${ }^{17-19}$; in contrast, human BMPC produce Ig in vitro for prolonged periods of time (at least two weeks), and are not susceptible to apoptosis through ligation of the death receptor CD95. ${ }^{18} 2021$ High expression of the CD38 molecule ( $\mathrm{CD} 38^{\mathrm{h}}$ phenotype) by all human $\mathrm{PC}^{22}$ has provided a tool for comparative phenotypic analysis of PC obtained from different territories. It reveals that BMPC exhibit features indicating a level of maturation higher than that shown by PC from inductive areas (tonsils, blood). ${ }^{23}$ Taken together, these findings support the view that PC present in the BM, the effector site for systemic humoral responses, have reached the highest survival state and functional capacities. The mechanisms that underlie migratory and maturative events giving rise to BMPC remain to be elucidated.

Less attention has generally been paid to PC responsible for the mucosal humoral immune response. Nevertheless, the number of PC generated by mucosal antigen stimulation is larger and they produce more Ig than PC induced in systemic responses. ${ }^{24} 25$ Thus following the arrival of antigens at a variety of mucosal associated lymphoid organs, antigen induced foci and activated germinal centres are rapidly developed. ${ }^{26}$ Examples of such organs are intestinal Peyer's patches and, in part, the tonsils, which collectively are considered inductive areas for mucosal humoral immune responses. It is thought that from these inductive areas antigen primed B cells migrate through the circulation into effector sites for mucosal humoral responses, the most prominent of which is the intestinal lamina propria (LP), where large numbers of PC committed to mucosal antigen responses are commonly found. ${ }^{27}$ These PC predominantly produce $\mathrm{Ab}$ of the $\operatorname{IgA}$ isotype which are mainly secreted across mucous membranes and whose function is essential for defence against the arrival of potentially invasive micro-organism at the mucosal surfaces. ${ }^{28}$ Recent experiments in mice show that maturation of certain intestinal IgA secreting PC, mostly committed to commensal bacteria, can occur from Bl lymphocytes, and these PC follow a $\mathrm{T}$ cell and lymphoid follicle independent pathway, apparently guided by interaction with LP stromal cells. ${ }^{29}{ }^{30}$ Evidence supporting this pathway in humans has not as yet been documented. In fact, the majority of human LPPC harbour heavily

Abbreviations: Ab, antibody; Blimp-1, B lymphocyte induced maturation protein 1 ; $B M$, bone marrow; $B S A P, B$ cell specific activation protein; CyC, Cy-Chrome; FITC, fluorescein isothiocyanate; LP, lamina propria; $\mathrm{mAb}$, monoclonal antibody; $\mathrm{MFI}$, mean fluorescence intensity PBS, phosphate buffered saline; PC, plasma cells; PE, phycoerythrin; RT-PCR, reverse transcriptase-polymerase chain reaction. 


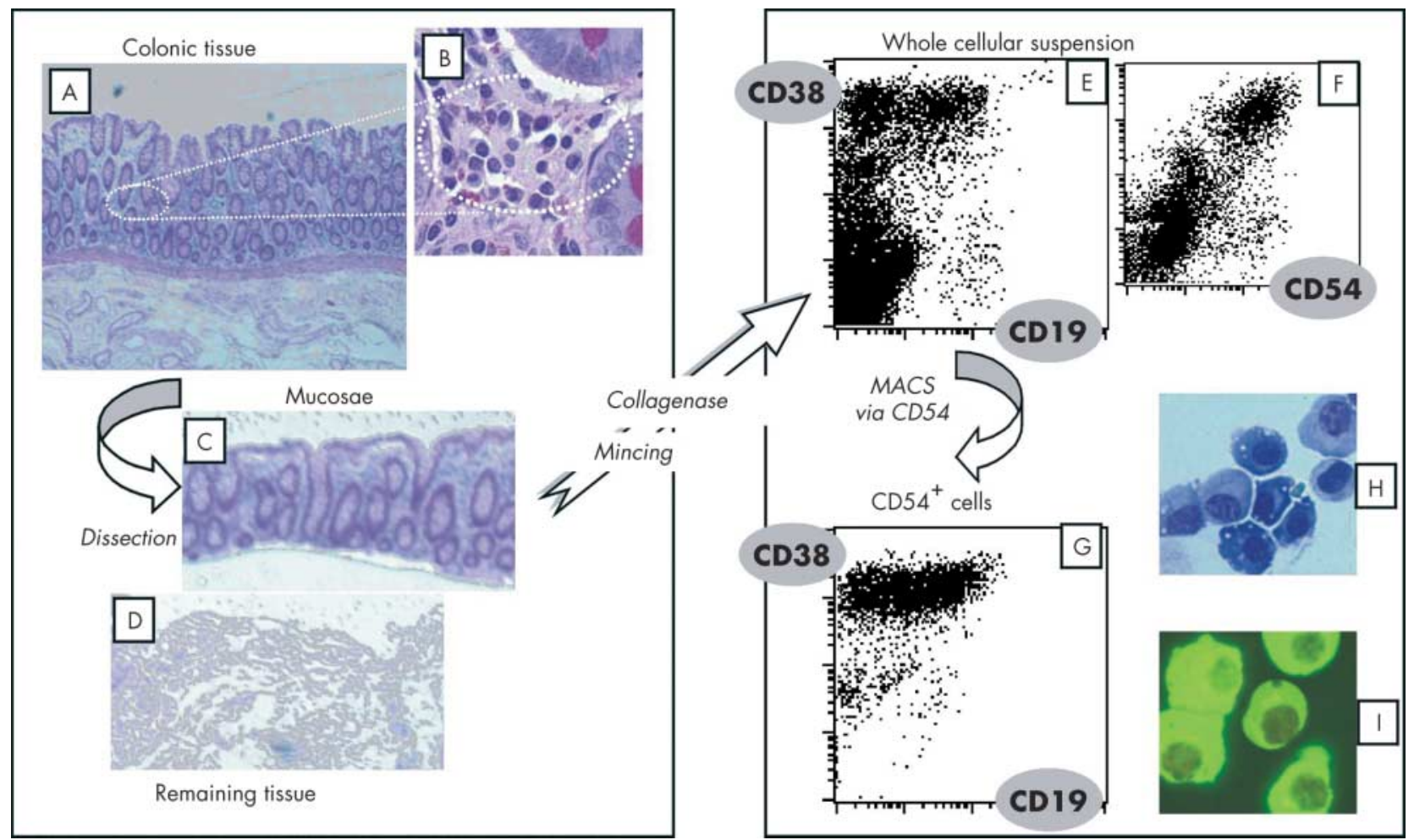

Figure 1 Purification of plasma cells (PC) from human colon lamina propria (LP). The figure shows a representative example of the consecutive steps followed in the protocol used for the purification of LPPC. (A) Haematoxylin-eosin staining of a colon wall section showing the different layers. (B) Giemsa staining of the LP area showing abundant PC. (C, D) Haematoxylin-eosin staining of the dissected mucosal layer (including epithelium and LP areas) and the remaining tissue, respectively. (E) LP cells were obtained by collagenase digestion of the mucosal layer (C) and studied using labelled monoclonal antibodies (mAb) and flow cytometry analysis. A dot plot analysis of the CD19/CD38 cell expression is shown: a cell subset of CD38 $8^{\mathrm{h}}$ CD 19 ${ }^{+/-}$can be observed. (F) Dot plot analysis of LP cells labelled for CD38 and CD54 showing the distinctive expression of CD54 by the LP CD38 ${ }^{\text {h }}$ cells. (G) Dot plot analysis of CD38 and CD 19 expression of LP CD54 selected cells showing the high degree of enrichment in $C D 38^{\mathrm{h}}$ cells obtained by this procedure. $(\mathrm{H}, \mathrm{I}) \mathrm{CD} 38^{\mathrm{h}}$ cells purified by CD54 immunomagnetic selection were identified as PC by Giemsa staining as well as by intracytoplasmic lgA staining of cytospin preparations, respectively.

mutated IgV genes, ${ }^{31}$ indicating that they have undergone strong antigen selection, a feature apparently restricted to the germinal centre pathway and characteristic of highly differentiated PC. ${ }^{112}$ Despite their importance, a wide phenotypic analysis of LPPC has not as yet been performed. In particular, it is not known whether LPPC share similarities with PC from the BM, the other well established PC effector site. Such a study has been hampered by the lack of a suitable method for LPPC isolation.

The present work reports a fast and effective method for purifying PC from human colon LP. This technique allowed us to confirm the correspondence between LP CD $38^{\mathrm{h}}$ cells and PC and to explore "ex vivo" functional capabilities of isolated LPPC, such as the kinetics of Ig secretion and CD95 ligation effect. In order to gain a deeper insight into the maturational stage of LPPC, their expression of several molecules was explored using labelled mouse monoclonal antibodies and flow cytometry analysis. These molecules include differentiation markers, and survival related and adhesion molecules. In addition, the presence of mRNA for the two relevant B cell transcription factors B lymphocyte induced maturation protein 1 (Blimp-1) and B cell specific activating protein (BSAP) was investigated. The results were compared with those of other PC populations, particularly those from BM.

\section{MATERIALS AND METHODS}

\section{Materials}

Cycloheximide, purified mouse IgG, and collagenase V were purchased from Sigma (St Louis, Missouri, USA). EDTA was provided by Pharmacia Biotech (Uppsala, Sweden). Purified mouse monoclonal antibody (mAb) against CD54, fluorescein isothiocyanate (FITC) labelled mAb against CD19 and CD44, phycoerythrin (PE) labelled mAb against CD19, CD20, CD31, CD49d, CD54, CD95, HLA-DR, and mouse IgG, Cy-Chrome $(\mathrm{CyC})$ labelled $\mathrm{mAb}$ against $\mathrm{CD} 38$, the corresponding isotypic negative controls, and PE labelled rat antimouse IgG mAb were purchased from Becton Dickinson (San Jose, California, USA). Apoptosis inducing mAb against CD95 (clone CH11) and PE labelled mAb against CD2 1 were provided by Coulter (Miami, Florida, USA). FITC labelled mAb against VS38c and $\mathrm{Bcl}-2$, FITC conjugated rabbit antihuman $\operatorname{IgA}$, and the intrastain fixation permeabilisation kit were from Dako (Glostrup, Denmark). Unconjugated and peroxidase conjugated goat $\mathrm{F}(\mathrm{ab})_{2}^{\prime}$ antihuman IgA and anti-human IgG used in ELISA were purchased from Biosource (Camarillo, California, USA). ACT- 1 mAb against the adhesion molecule $\alpha 4 \beta 7$ was kindly provided by Dr Ortíz de Landázuri (Hospital de la Princesa, Madrid, Spain). Goat antimouse IgG bound to magnetic microbeads, selection columns of $\mathrm{LS}^{+}$type, and midiMACS magnet were obtained from Miltenyi Biotec (Auburn, California, USA). Ninety six well flat bottomed culture plates and U96 Maxisorp plates used for ELISA were provided by Nunc (Roskilde, Denmark)

\section{Isolation of colon lamina propria (LP), tonsil, and bone marrow (BM) cells}

Human colonic tissue (fig 1A, B) was obtained from normal areas of surgically resected specimens. The mucosal layer was mechanically dissected out (fig IC) from the colon wall sample using two scalpels. This process released a fine tissular sheet including the epithelium and LP (fig ID). After washing in phosphate buffered saline (PBS), the layer was minced in a 
Petri dish and treated with collagenase V ( $1 \mathrm{mg} / \mathrm{ml}$ in RPMI1640) for 15 minutes at $37^{\circ} \mathrm{C}$ in a shaking bath. The reaction was stopped by adding culture medium (see below), and the resulting cell suspension was centrifuged at low speed $(20 \mathrm{~g}$, three minutes) to separate undigested tissue pieces. The supernatant containing released cells was collected, centrifuged at $400 \mathrm{~g}$ for seven minutes, and washed twice in culture medium. The resulting cell fraction will be referred as to LP cells. BM and tonsil PC were purified as previously reported. ${ }^{23}$ Approval was obtained from the Institutional Review Board (Comité Etico, Hospital Universitario Puerta del Mar) for these studies.

\section{Isolation of CD54 ${ }^{+}$cells from LP cell preparations}

CD54 ${ }^{+}$cells were purified from the LP cell fraction by an immunomagnetic technique. Briefly, cells (up to $100 \times 10^{6}$ cells/ $\mathrm{ml})$ were incubated with anti-CD54 mAb $(10 \mu \mathrm{g} / \mathrm{ml})$ in $2 \mathrm{mM}$ EDTA $0.5 \%$ bovine serum albumin in PBS for 10 minutes in the dark at $4^{\circ} \mathrm{C}$. After two washes in the same buffer, cells (up to $100 \times 10^{6}$ cells $/ \mathrm{ml}$ ) were incubated with goat antimouse magnetic micro beads, according to the manufacturer's instructions. After two washes, cells were resuspended (up to $50 \times 10^{6} \mathrm{cell} / \mathrm{ml}$ ) in the buffer. A separation column was placed in the magnet and $500 \mu \mathrm{l}$ of buffer were applied at the top of the column and allowed to run through, the effluent being discarded. The cell suspension was pipetted onto the column and allowed to run through. The effluent was collected as negative cell fraction. The column was washed three times with $3 \mathrm{ml}$ of buffer and the effluents included in the negative cell fraction. At this point, the column was removed from the magnet, $5 \mathrm{ml}$ of buffer were applied at its top, and cells were firmly flushed out, using the supplied plunger. The effluent was collected as the positive cell fraction ( $\mathrm{LP} \mathrm{CD} 54^{+}$cells).

\section{Cell culture and IgA ELISA}

LP CD $54^{+}$cells were adjusted to $0.5 \times 10^{6}$ cells $/ \mathrm{ml}$ in a culture medium consisting of RPMI 1640 supplemented with 10\% fetal calf serum, L-glutamine ( $10 \mathrm{mM})$, and gentamycin $(0.05$ $\mathrm{mg} / \mathrm{ml}$ ), and were cultured in 96 well plates in a final volume of $250 \mu \mathrm{l} /$ well at $37^{\circ} \mathrm{C}$ with $5 \% \mathrm{CO}_{2}$. In some experiments cells were cultured in the presence of the apoptosis inducing anti$\mathrm{CD} 95 \mathrm{mAb} \mathrm{CH} \mathrm{l}$ at $400 \mathrm{ng} / \mathrm{ml}$. After four, seven, and 14 days, cell free supernatants were collected, and IgA secretion was tested by enzyme linked immunoabsorbent assay in microtitre plates, as previously reported. ${ }^{16}$

\section{Cell staining and flow cytometry}

For three colour labelling experiments, $200 \mu \mathrm{l}$ of LP and BM cells (at $5 \times 10^{6} \mathrm{cell} / \mathrm{ml}$ ) were incubated with optimal concentrations of a variety of labelled mAb (see below) for 20 minutes in the dark at $4^{\circ} \mathrm{C}$. After two washes, cell phenotype was analysed by flow cytometry. To detect Bcl-2 and VS38c (both intracellular molecules), a fixation/permeabilisation kit (Dako) was used after surface staining, following the manufacturer's instructions. $\alpha 4 \beta 7$ expression was detected by a direct/indirect staining technique. Cells were incubated with mouse ascites containing ACT- 1 mAb (1/100 dilution), washed, and stained with a PE conjugated rat antimouse $\mathrm{mAb}$. After washing, cells were incubated with purified mouse IgG ( $1 \mu \mathrm{g} / \mathrm{ml}$ ) for 30 minutes to block any free binding site of the secondary mAb. After two washes in PBS, cells were stained with FITC labelled $\mathrm{mAb}$ against $\mathrm{CD} 19$ and $\mathrm{CyC}$ labelled $\mathrm{mAb}$ against CD38. All incubation and washing steps were performed under the conditions indicated above. FACS analysis was performed on a FACScalibur cytometer (Becton Dickinson) equipped with an air cooled argon ion laser emitting 15 $\mathrm{mW}$ at $488 \mathrm{~nm}$. The instrument was equipped with three fluorescence detector photomultiplier tubes, with green fluorescence (FITC) being collected through a 530/30 $\mathrm{nm}$ bandpass, red/orange (PE) through a 585/42 $\mathrm{nm}$ bandpass, and red
(CyC) through a $650 \mathrm{~nm}$ longpass filter. Cell analysis was performed with Cellquest software (Becton Dickinson). Light scatter signals were recorded in linear mode and fluorescence signals in logarithmic mode. PC present in the LP and BM mononuclear cell fractions were identified by their $\mathrm{CD} 38^{\mathrm{h}}$ expression profile in a CD19/CD38 dot plot. The third fluorescence was used to explore PC expression of differentiation markers (CD19, CD20, VS38c, and HLA-DR), survival factors (CD95 and Bcl-2), and adhesion molecules (CD21, CD31, CD44, CD49d, CD54, and $\alpha 4 \beta 7)$. Data from 2000-5000 CD38 cells/sample were collected, and the percentage as well as mean fluorescence intensity (MFI) of $\mathrm{CD} 38^{\mathrm{h}}$ cells positive for each analysed molecule were monitored. Results are expressed as mean (SEM) of a variety of experiments. Statistical analysis was carried out by using the Student's $t$ test. Differences were considered significant when $\mathrm{p}<0.05$.

\section{Detection of the B cell transcription factors Blimp-1 and BSAP}

The presence of transcripts of Blimp-1 and BSAP was investigated in human highly purified PC from tonsils (T-PC), LP (LPPC), and BM (BMPC) by reverse transcriptase- polymerase chain reaction (RT-PCR). To this end, total RNA from each cellular fraction was purified using the acid-guanidinethiocyanate-phenol-chloroform method.

After a DNAse I treatment, first strand cDNA copies were synthesised using avian myeloblastosis virus reverse transcriptase (Promega, Barcelona, Spain) with random hexamers (pd(N6); Amersham Pharmacia Biotech, Barcelona, Spain) for priming. Then, PCR was performed with the following oligonucleotide primers: for Blimp-1 sense primer 5'- ATGCGGATATGAC TCTGTGGA-3' and antisense primer $5^{\prime}$ CTCGGTTGCTTTAGACTGCTC-3', for BSAP (sense) 5' CAGCATAGTGTCCACTGGCT-3' and (antisense) $5^{\prime}$ CCTGTCAGCGTCGGTGCTGA-3'. CDNA ( $3 \mu \mathrm{l})$ was amplified in a PTC-100 MJ Thermocycler (MJ-Research Inc., Waltham, Massachusetts, USA) using each primer and Taq DNA polymerase (Biotaq, Bioline Ltd, UK). The cycler conditions for Blimp-l and BSAP consisted of a denaturing step at $94^{\circ} \mathrm{C}$ for one minute, an annealing step at $65^{\circ} \mathrm{C}$ for one minute, and an amplification step at $72^{\circ} \mathrm{C}$ for one minute, for 35 cycles, and a final additional amplification step at $72^{\circ} \mathrm{C}$ for seven minutes. The amplified products were analysed on a $1.2 \%$ agarose gel containing ethidium bromide and visualised by UV light illumination. The quantity of $\beta$-actin cDNA was evaluated using a

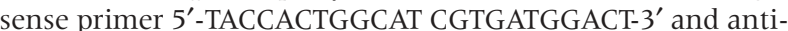
sense primer $5^{\prime}$ - CGTCACACTTCATGATGGAG-3' as CDNA internal controls. The cycler conditions for $\beta$ - actin were as above, except that the annealing temperature was $62^{\circ} \mathrm{C}$ and only 30 cycles were performed.

\section{Histology and plasma cell identification}

Whole colon tissue and fragments resulting from dissecting out the mucosal layer were processed for histological analysis. In brief, samples were formalin fixed, paraffin embedded, and stained with haematoxylin and eosin. Whole colon tissue slides were additionally stained by a Giemsa technique to identify PC "in situ". LP CD54 cells $\left(0.1 \times 10^{6}\right.$ cells in $100 \mu \mathrm{l}$ of PBS) were cytocentrifuged on slides and also stained (Giemsa), and cells with PC morphology were determined. Cells containing intracytoplasmic IgA were detected on similar cytospin cell preparations by a direct immunofluorescence technique. Positive cells were identified by fluorescence microscopy.

\section{RESULTS}

Purification of human colon lamina propria plasma cells (LPPC)

The first aim of this study was to design a protocol to purify human LPPC in a period of time that preserved PC and allowed their functional assessment. A colonic mucosal layer 

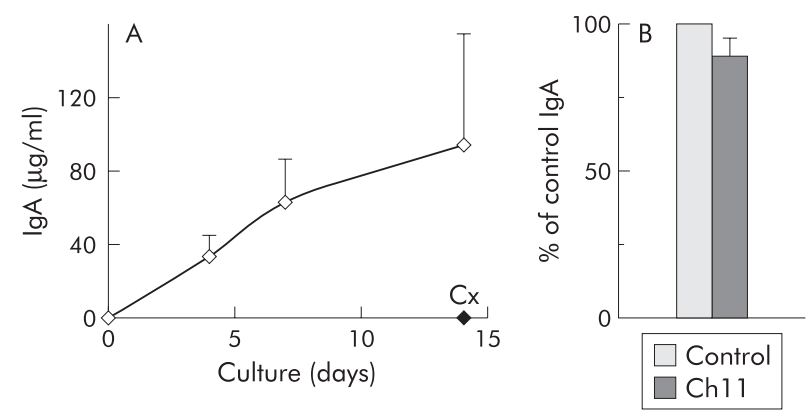

Figure 2 In vitro lgA secretion by lamina propria (LP) CD38 $8^{\mathrm{h}}$ cells. (A) Purified LP CD38 ${ }^{\mathrm{h}}$ cells were cultured for the indicated periods of time, and the quantity of $\lg A$ secreted into the supernatant was assessed by enzyme linked immunoabsorbent assay. Cycloheximide $(C \times 10 \mu \mathrm{g} / \mathrm{ml})$ was added to certain cultures. Results are expressed as $\mu \mathrm{g} / \mathrm{ml}$ of $\lg A$, and represent the mean (SEM) of four experiments. (B) LP CD38 ${ }^{\text {h }}$ cells were cultured in the absence (Control) and presence (Ch 1 1) of the anti-CD95 mAb CHI 1 (400 ng/ml) for seven days, and $\lg \mathrm{A}$ secreted into the supernatant was determined. The results are expressed as the percentage of control $\lg A$ secretion, and represent the mean (SEM) of six experiments.

that included epithelium and LP (fig IC) was obtained and a cell suspension was released after a brief collagenase treatment (LP cell fraction). The presence of PC in this cell preparation was investigated by cell staining with labelled $\mathrm{mAb}$, followed by flow cytometry analysis. It is well established that human PC express high levels of CD38 molecules on their surface $\left(\mathrm{CD} 38^{\mathrm{h}}\right) \cdot{ }^{17-22}$ As can be seen in fig $1 \mathrm{E}$, a subset of LP cells exhibiting the phenotype CD38
$\mathrm{CD}^{+1 /-}$ could be clearly detected in the $\mathrm{CD} 38 / \mathrm{CD} 19$ dot plot. This cell subset accounted for 21.5 (2)\% (mean (SEM); $\mathrm{n}=16$ ) of the total LP cell fraction which, in addition to this putative PC population, consisted of B and T lymphocytes and epithelial cells (data not shown). Subsequently, LP cells were triple stained (for CD38, CD19, and a third molecule) in search of a marker present on $\mathrm{CD} 38^{\mathrm{h}}$ cells that was suitable for use in an immunomagnetic purification strategy. Among all the molecules tested, CD54 was the co- expressed marker that permitted the clearest distinction of $\mathrm{CD} 38^{\mathrm{h}}$ cells from the rest of the LP cells (fig IF). Accordingly, immunomagnetic selection by the presence of CD54 was carried out in LP cell populations. Selected $\mathrm{LP} \mathrm{CD} 54^{+}$cells were all positive for expression of the CD54 molecule, as expected (data not shown). Subsequently, the LP CD $54^{+}$cell fraction was stained for $\mathrm{CD} 38$ and CD19 showing a highly purified $\mathrm{CD} 38^{\mathrm{h}}$ population (fig $1 \mathrm{G}$ ). $\mathrm{CD} 54^{+}$selected cells consisted almost entirely of CD38 ${ }^{\text {h }}$ cells (94.4 ( 1$) \%$; mean $\left.(\mathrm{SEM}) ; \mathrm{n}=4\right)$. In addition, LP $\mathrm{CD} 4^{+}$selected cells contained intracytoplasmic IgA (fig II) and could be morphologically identified as typical PC ( fig $1 \mathrm{H}$ ). LPPC purification by a direct $\mathrm{CD}_{3} 8^{+}$immunomagnetic selection procedure yielded significantly inferior results (data not shown).

\section{"Ex vivo" spontaneous Ig secretion by isolated LPPC: kinetics and anti-CD95 mAb effect}

In vitro Ig secreting capacity of LPPC was examined next. Isolated LPPC were cultured for four, seven, and 14 days, culture supernatants were harvested at the end of the culture period, and IgA secretion was quantified by ELISA. As shown in fig 2A, LPPC linearly secreted IgA for 14 days. IgA secretion was
A
CD20

LP

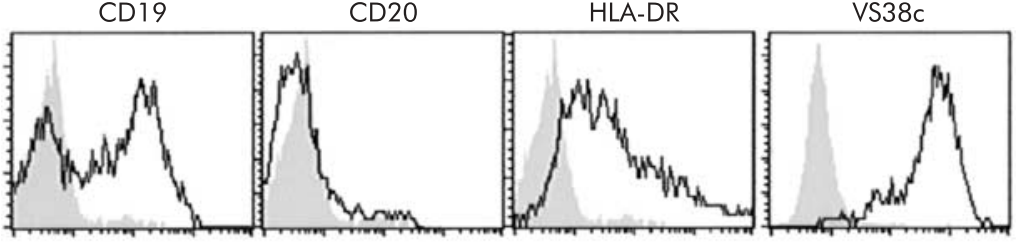

$\mathrm{BM}$

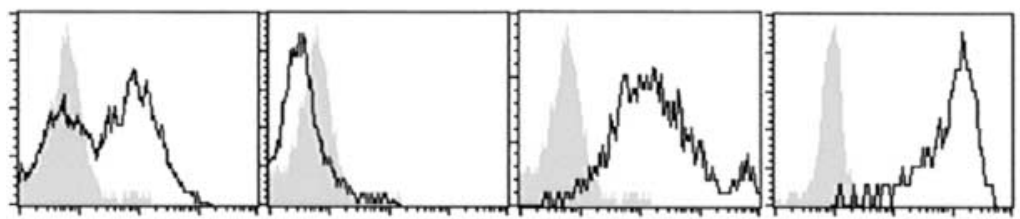

CD95
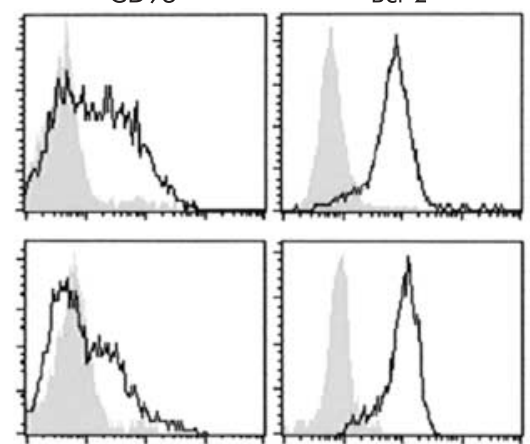

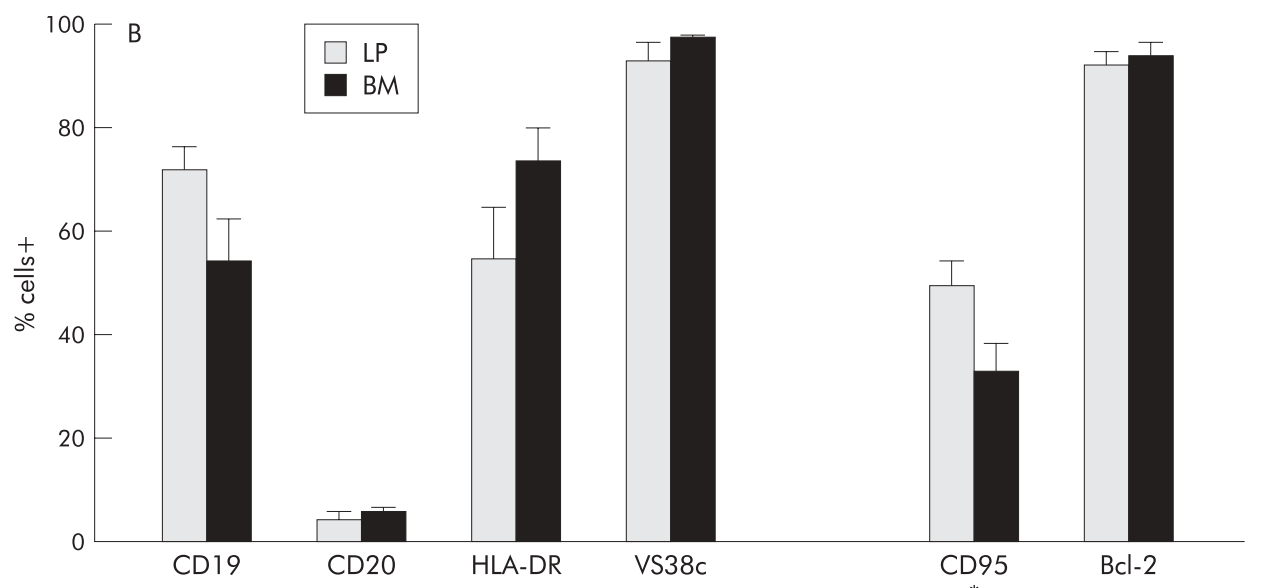

Figure 3 Differentiation and survival molecule expression by lamina propria (LP) and bone marrow (BM) plasma cells (PC). (A) Representative example (top series of histograms) of LPPC and BMPC (CD38 cells) expression of several differentiation molecules (CD19, CD20, HLA-DR, and VS38c) and survival associated molecules (CD95, Bcl-2). Negative control histograms are shown in grey. (B) Mean (SEM) of several experiments $(n \geqslant 5)$ for each marker, expressed as a percentage of positive $P C$. ${ }^{*} p<0.05$. 


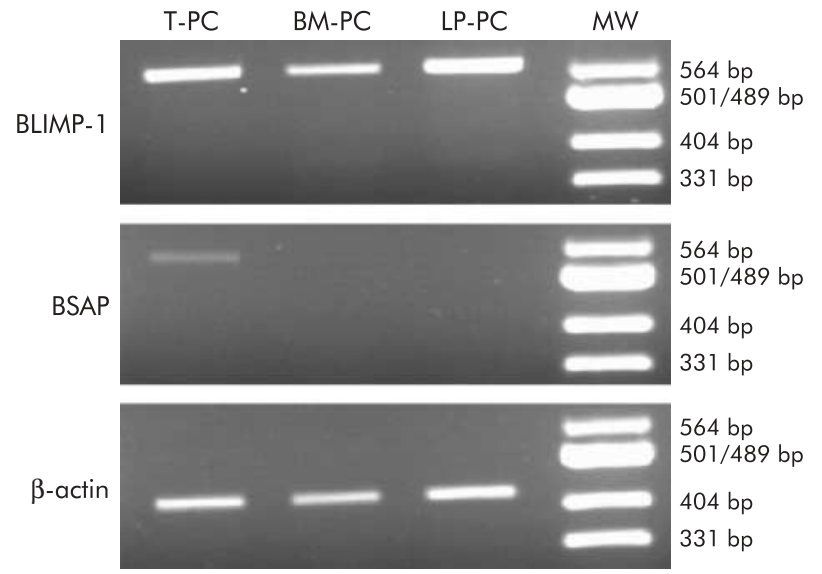

Figure 4 Messenger RNA for PRDI-BF 1/B lymphocyte induced maturation protein 1 (Blimp 1) and B cell specific activation protein (BSAP) expression levels in different plasma cell (PC) populations. Relative amounts of Blimp-1 and BSAP mRNA were determined by reverse transcriptase-polymerase chain reaction (RT-PCR) from highly purified tonsil PC (T- PC), lamina propria PC (LP-PC), and bone marrow PC (BM-PC). RT-PCR using $\beta$-actin mRNA sequences served as an internal standard. As a molecular weight marker, a Msp I digestion of pUC19 was used (MW). Data are representative of the results of three experiments using different donors.

actively carried out by the purified LPPC as addition of the protein synthesis inhibitor cycloheximide reduced substantially this capacity. In addition, inclusion of the apoptosis inducing anti-CD95 mAb CHll into LPPC cultures did not provoke a significant decrease in their spontaneous IgA secretion (fig 2B).

\section{Comparative analysis of differentiation markers, death/survival molecules, and B cell transcription factor expression by PC from LP and BM}

Expression of several B cell differentiation markers (CD19, CD20, HLA-DR, and VS38c), the death receptor CD95, and the antiapoptotic molecule Bcl-2 was assessed by using labelled $\mathrm{mAb}$ and flow cytometry analysis in PC populations from both BM and LP. BM mononuclear cell and LP cell populations were triple stained, and PC were identified as $\mathrm{CD} 38^{\mathrm{h}}$ cells in a CD38/CD19 dot plot. Figure 3 compares expression of these molecules by PC from LP and BM, showing a representative example of the obtained histograms (fig 3A), and a summary of several experiments expressed as percentage of positive PC for each molecule (fig 3B). As can be seen, all differentiation markers were similarly expressed by PC from both LP and BM. Thus CD19 showed a double population (negative/positive), CD20 was completely negative, HLA-DR exhibited a broad spectrum of intensity levels ranging from intermediate to negative expression, and VS38c, an endoplasmic reticulum protein described as a good marker for $\mathrm{PC}^{32}$ was highly expressed by both PC populations. For expression of the death receptor $\mathrm{CD} 95$, there was a mixture of positive and negative PC in both territories. In contrast, BMPC and LPPC were clearly positive for the antiapoptotic molecule $\mathrm{Bcl}-2 .{ }^{33}$ In addition, the presence of transcripts for BSAP and Blimp-1 B cell specific transcription factors ${ }^{34-37}$ was investigated in highly purified tonsil PC, BMPC, and LPPC by RT-PCR (fig 4).

\section{Comparative analysis of adhesion molecule expression by LPPC and BMPC}

It is now clear that the profile of adhesion molecules expressed by a given cell plays an important role in determining its entry and attachment to specific microenvironments. Accordingly,
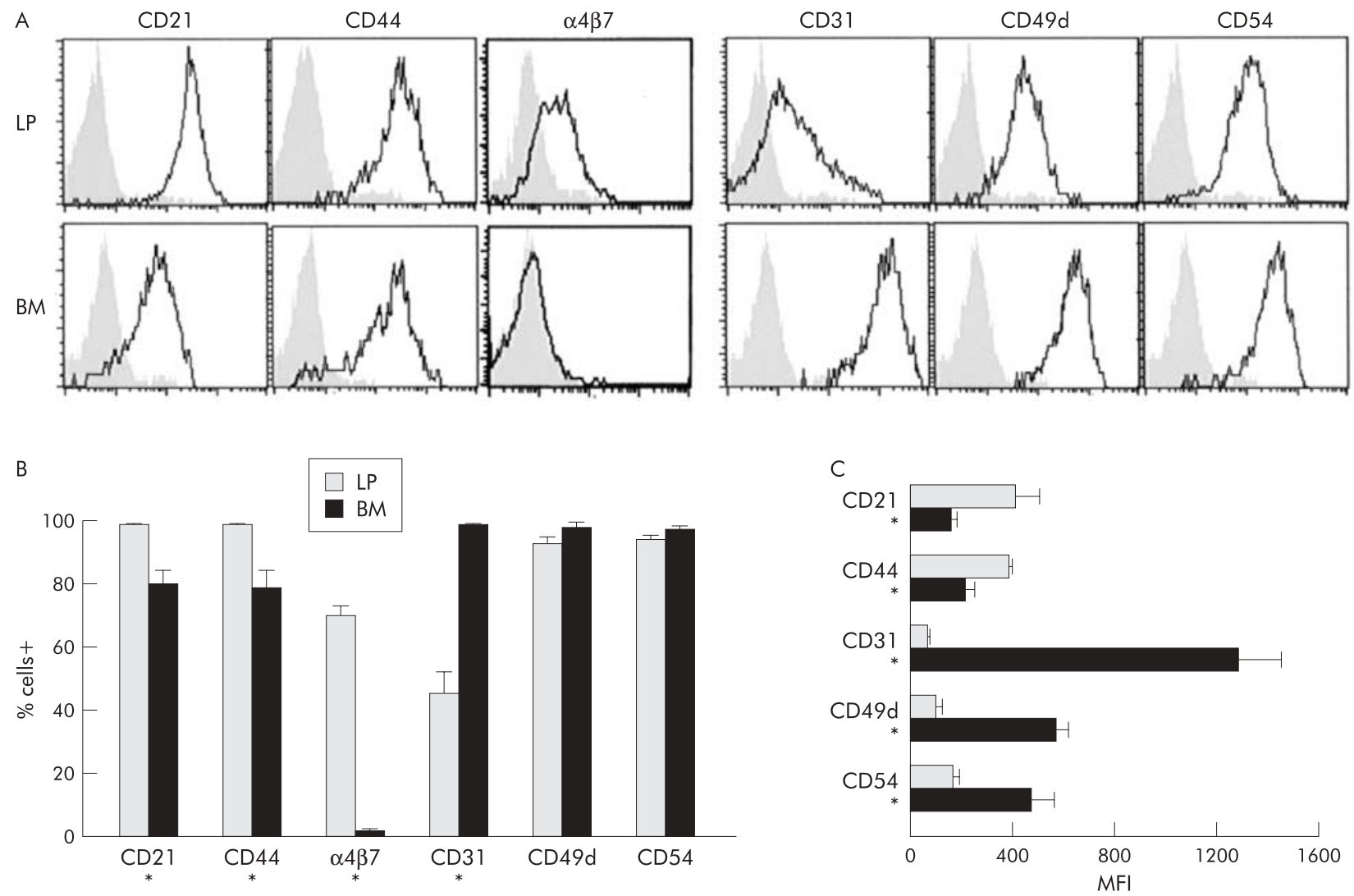

Figure 5 Adhesion molecule expression by lamina propria (LP) and bone marrow (BM) plasma cells (PC). (A) Representative example (upper series of histograms) of LPPC and BMPC (CD38 ${ }^{\mathrm{h}}$ cells) expression of several adhesion molecules (CD21, CD44, $\alpha 4 \beta 7, C D 31, \mathrm{CD} 49 \mathrm{~d}$, and CD54). Negative control histograms are shown in grey. (B) Mean (SEM) of several experiments ( $\geqslant 55$ ) for each marker expressed as a percentage as well as mean fluorescence intensity (MFI) (C) of positive PC. ${ }^{\star} p<0.05$. 
BMPC and LPPC expression of several adhesion molecules (CD21, CD44, $\alpha 4 \beta 7, C D 31, C D 49 d$, and CD54) was also evaluated using labelled $\mathrm{mAb}$ and flow cytometry analysis. Figure $5 \mathrm{~A}$ shows a representative example of this study. The results obtained in all experiments are summarised in fig 5, and are represented as a percentage as well as the MFI of positive PC for each molecule under analysis (fig 5B, 5C, respectively). Although most of these markers were positive in both PC populations, the intensity of their expression differed greatly. Thus CD21 and CD44 staining was twice as high on PC from LP compared with PC from BM. $\alpha 4 \beta 7$ was positive on LPPC, as previously reported, ${ }^{38}$ but totally negative on BMPC. In contrast, CD31, CD49d, and CD54 staining was more pronounced on BMPC than on LPPC. The difference was specially notable for CD31 and CD49d (20-fold and 10-fold increase, respectively).

\section{DISCUSSION}

Current methods of processing colon tissue to obtain LP cells generally consist of several washing steps and an overnight collagenase incubation to release lymphoid cells from LP. This procedure is time consuming and may cause damage to the functional capacities of LPPC. Accordingly, an initial aim of this study was to design a method for isolating LPPC that preserves cell function. The mechanical separation of a thin layer that only included the mucosal epithelium and the LP enabled the collagenase digestion period to be reduced to 15 minutes, which was sufficient for release of most LP cells. From this LP cell fraction, $\mathrm{CD} 38^{\mathrm{h}}$ cells were highly purified by positive immunomagnetic selection through CD54, a marker that is distinctively expressed by LP CD $38^{\mathrm{h}}$ cells. The correspondence between LP CD $38^{\text {h }}$ cells and PC was directly demonstrated by both morphology and bright intracytoplasmic anti- IgA staining criteria. Therefore, this present procedure is efficient for obtaining highly purified human colonic LPPC.

Isolated LPPC spontaneously produced large quantities of $\operatorname{IgA}$ in vitro. Lower amounts of IgM were also secreted into these supernatants and, in some experiments, marginal quantities of IgG secretion could be detected (data not shown). On average, IgA secretion accounted for $80 \%$ or more of the total Ig produced in vitro, a finding that is in agreement with the isotype distribution described for colon Ig secreting cells by immunohistochemical techniques. ${ }^{28}$ LPPC spontaneously produced IgA in vitro in an active way, and did so linearly for at least two weeks. This prolonged kinetics of IgA secretion clearly distinguishes LPPC from PC occurring in inductive territories such as the tonsils, lymph node, and blood, ${ }^{13-16}$ and assimilates them with BMPC, which also show prolonged kinetics of Ig secretion in vitro. ${ }^{2021}$ On a per cell basis, the LPPC IgA secretion rate can be estimated as approximately $10^{7}$ molecules per hour, which is similar to that calculated for PC by other authors. ${ }^{17} 20$ Thus LPPC isolated by this method retain their proper functional capacities.

The comparative phenotypic study of LPPC and BM PC revealed the existence of additional similarities between these cells regarding expression of a variety of differentiation markers. Both populations were $\mathrm{CD} 19^{+} \digamma \mathrm{CD} 20^{-}$and HLA-DR ${ }^{\text {low }}$, and stained brightly for VS38c, denoting a well developed endoplasmic reticulum, as expected from their capacity for secreting Ig at a high rate. It is interesting to remark that the pattern of differentiation molecules shared by BMPC and LPPC markedly differs from that observed on human PC obtained in inductive areas such as the tonsils, as these latter are $\mathrm{CD} 20^{+} \mathrm{CD} 19^{+} \mathrm{HLA}-\mathrm{DR}^{\text {high }}$, and show lower levels of VS38c expression, ${ }^{23}$ a finding that emphasises their relative functional immaturity.

Analysis of CD95 expression showed that an average of 50\% of LPPC and 30\% of BMPC carried the death receptor. Despite this fact, CD95 ligation in both PC populations had no effect on Ig secretion in culture (Medina and colleagues ${ }^{18}$ and the present study), indicating that they were not susceptible to apoptosis by this signalling mechanism. It is well established that mere expression of CD95 does not confer susceptibility to apoptosis on CD95 signalling. ${ }^{39}$ Accordingly, failure in inducing apoptosis through CD95 ligation as well as partial CD95 expression exhibited by these PC may be explained as either a residual temporary presence of this death receptor or as an inefficient or blocked death inducing signalling complex. In addition, LPPC and BMPC exhibited a high level of expression of Bcl-2, a well known antiapoptotic protein. ${ }^{33}$ These findings are clearly in contrast with those reported for human tonsils and blood PC that show higher levels of CD95, undergo apoptosis on CD95 cross linking, and contain lower amounts of Bcl-2.2. Thus low expression of CD95 and resistance to apoptosis via this receptor, together with high expression of Bcl-2, may be associated with the prolonged kinetics of Ig secretion in vitro shown by PC present in effector sites of the humoral immune response, such as BM and colon LP.

BSAP, the product of the pax 5 gene, is a B cell specific transcription factor that plays an essential role in B cell lineage commitment ${ }^{34}$ and development. ${ }^{40}$ In fact, BSAP expression is only lost at the PC stage. ${ }^{41}$ In contrast, the transcription factor Blimp-1 is expressed only in later B cell differentiation stages $^{35}$ where this factor appears to control a variety of aspects of the maturation programme of PC. ${ }^{35}$ Enforced expression of BSAP inhibits the final differentiation of B lymphocytes into PC. ${ }^{42}$ It has been shown previously that the transition to terminally differentiated PC in humans is associated with the presence of the transcription factor Blimp- 1 and loss of the transcription factor BSAP. ${ }^{23}$ Our results reveal that LPPC as well as BMPC expressed such a profile of transcription factors. In contrast, tonsil PC continue to express BSAP, suggesting again that PC from inductive areas appear to be less mature. Taken together, all of the results reported here establish a clear distinction between BMPC and LPPC on the one hand and PC present in inductive organs on the other, in that the latter PC appear less differentiated and exhibit a propensity to undergo apoptosis whereas the former PC show higher differentiation, survival, and functional capacity. The mechanisms involved in producing these differences remain to be elucidated.

In spite of the similarities demonstrated for human BMPC and LPPC, analysis of their respective profiles of adhesion molecule expression reveals marked differences. Thus CD21 and CD44 expression was brighter on LPPC than on BMPC. Moreover, expression of the integrin $\alpha 4 \beta 7$ was only found on LPPC. In contrast, CD31, CD49d, and CD54 showed a higher presence on BMPC than on LPPC. Taken together, these findings demonstrate the occurrence of specific patterns of adhesion molecule expression for each PC population, probably related to the ligands occurring in their particular microenvironment. High expression of CD49d and CD31 by BMPC probably indicates a role for these two molecules in the homing and attachment of PC to BM specific niches. The integrin $\alpha 4 \beta 7$ plays a key role in the homing of lymphoid cells to mucosal tissues including the LP, as has been demonstrated in in vivo animal models ${ }^{43-45}$ and, more indirectly, in human in vitro adhesion assays. ${ }^{46}$ Little is known of the specific adhesion molecules used by circulating PC precursors to migrate into the LP. In this respect, B cell blasts detected in microlymphatic vessels of Peyer's patches are $\alpha 4 \beta 7^{+},{ }^{47}$ and Ab secreting cells released to the circulation after oral vaccination are exclusively detected in the $\alpha 4 \beta 7^{+}$cell fraction. ${ }^{48}$ These observations, together with the finding that LPPC are also $\alpha 4 \beta 7^{+}$, appear to indicate that this integrin might act in the recognition of mucosal endothelium by LPPC precursors. Expression of $\alpha 4 \beta 7$ by LPPC was weak in comparison with that of CD21, CD44, CD49d, or CD54. Therefore, adhesion between PC and the LP microenvironment could depend on some of these molecules, or perhaps others not tested, rather than on $\alpha 4 \beta 3$. Further work will be required to ascertain these possibilities. 
In summary, LPPC and BMPC have apparently reached the same terminal differentiation stage within the PC compartment, as can be deduced from their identical Ig secreting and survival capacity, and their common expression of differentiation markers. These characteristics seem to define PC occurring in effector areas of the humoral immune response (BM and LP), and distinguish them from those PC present in inductive territories. Nevertheless, BMPC and LPPC differ markedly in their adhesion molecules, in relation to their different localisation requirements. This study describing the phenotype and functional capabilities of normal PC from human intestinal LP could be helpful in the analysis of PC in intestinal diseases with an altered humoral immune response.

\section{ACKNOWLEDGEMENT}

We thank Dr J Martorell and Dr J Pérez-Requena for help in the histological studies. The study was supported by grant 01/1590 from Fondo de Investigaciones Sanitarias, Spain.

\section{Authors' affiliations}

F Medina, C Segundo, A Campos-Caro, I Salcedo, A García-Poley, J A Brieva, Servicio de Inmunología and Unidad de Investigación, Hospital Universitario Puerta del Mar, Cádiz, Spain

\section{REFERENCES}

1 Jacob J, Kassir R, Kelsoe G. In situ studies of the primary immune response to (4- hydroxy-3-nitrophenyl) acetyl. I. The architecture and dynamics of responding cell populations. J Exp Med 1991;173:116575

2 Liu Y-J, Zhang J, Lane PJ, et al. Sites of specific B cell activation in primary and secondary responses to $T$ cell-dependent and $T$ cell-independent antigens. Eur J Immunol 1991;21:2951-62.

3 Van Rooijen N. Direct intrafollicular differentiation of memory B cell into plasma cells. Immunol Today 1990;11:154-7.

4 Benner R, Hijmans W, Haaijman JJ. The bone marrow: the major source of serum immunoglobulins, but still a neglected site of antibody formation. Clin Exp Immunol 1981;46:1-8.

5 Koch G, Osmond DG, Julius MH, et al. The mechanism of thymus-dependent antibody formation in bone marrow. J Immunol $1981 ; 126: 1447-51$

6 MacMillan R, Longmire RL, Yelemosky R, et al. Immunoglobulin synthesis by human lymphoid tissues: normal bone marrow as a major site of $\lg G$ production. J Immunol 1972;109:1386-94.

7 Ho F, Lortan J, MacLennan I, et al. Distinct short-lived and long-lived antibody- producing cell populations. Eur J Immunol 1986;16:1297301 .

8 Slifka M, Antia R, Whitmire J, et al. Humoral immunity due to long-lived plasma cells. Immunity 1998;8:363-72.

9 Manz R, Thiel A, Radbruch A. Lifetime of plasma cells in the bone marrow. Nature 1997;388:133-4

10 Smith K, Hewitson T, Nossal G, et al. The phenotype and fate of the antibody-forming cells of the splenic foci. Eur J Immunol 1996:26:444-8

11 Smith K, Light A, Nossal G, et al. The extent of affinity maturation differs between the memory and antibody-forming cell compartments in the primary immune response. Embo J 1997; 16:2996-3006.

12 Takahashi Y, Dutta P, Cerasoli D, et al. In situ studies of the primary immune response to (4-hydroxy-3-nitrophenyl) acetyl. V. Affinity maturation develops in two stages of clonal selection. J Exp Med 1998; 187:885-95.

13 Stevens R, Macy E, Morrow C, et al. Characterization of a circulating subpopulation of spontaneous antitetanus toxoid antibody producing $B$ cells following in vivo booster immunization. J Immunol 1979;122:2498-504

14 De la Sen ML, García-Alonso A, Brieva JA. Human B lymphocytes capable of spontaneous Ig production in short-term cultures: characterization in the circulation and lymphoid tissues. Cell Immunol 1986;98:200-10

15 Muñoz J, Insel R. In vivo human antibody production to the hemophilus influenzae type b capsular polysaccharide. J Immunol 1987; 139:2026-31.

16 Brieva JA, Roldan E, Rodriguez C, et al. Human tonsil blood and bone marrow in vivo-induced $B$ cells capable of spontaneous and high-rate immunoglobulin secretion in vitro: differences in the requirements for factors and for adherent and bone marrow stromal cells, as well as distinctive adhesion molecule expression. Eur J Immunol 1994;24:362-6.

17 Merville P, Dechanet J, Desmouliere A, et al. Bcl-2+ tonsillar plasma cells are rescued from apoptosis by bone marrow fibroblasts. J Exp Med 1996;183:227-36.

18 Medina F, Segundo C, Rodriguez C, et al. Regulatory role of CD95 ligation on human $B$ cells induced in vivo capable of spontaneous and high-rate Ig secretion. Eur J Immunol 1997;27:700-6.
19 Jego G, Robillard N, Puthier D, et al. Reactive plasmacytoses are expansions of plasmablasts retaining the capacity to differentiate into plasma cells. Blood 1999;94:701-12.

20 Hibi T, Dosch HM. Limiting dilution analysis of B cell compartment in human bone marrow. Eur J Immunol 1986; 16:139-45.

21 Roldan E, Garcia-Pardo A, Brieva J. VLA-4 fibronectin interaction is required for the terminal differentiation of human bone marrow cells capable of spontaneous and high rate immunoglobulin secretion. J Exp Med 1992:175:1739-47.

22 Bhan A, Nadler LM, Stashenko P, et al. Stages of B cell differentiation in human lymphoid tissue. J Exp Med 1981;154:737-49

23 Medina F, Segundo C, Campos-Caro A, et al. The heterogeneity shown by human plasma cells from tonsil, blood and bone marrow reveals graded stages of increasing maturity, but local profiles of adhesion molecule expression. Blood 2002:99:2154-61.

24 Tseng J. Transfer of lymphocytes of Peyer's patches between immunoglobulin allotype congenic mice: repopulation of the lgA plasma cells in the gut lamina propria. J Immunol 1981;127:2039-43.

25 Brandtzaeg P, Halstensen TS, Kett K, et al. Immunobiology and immunopathology of human gut mucosa: humoral immunity and intraepithelial lymphocytes. Gastroenterology 1989;97:1562-84

26 Cebra JJ, Shroff KE. Peyer's patches as inductive sites for $\lg \mathrm{A}$ commitment. In: Ogra PL, ed. Handbook of mucosal Immunology. San Diego: Academic Press, 1994:151-8

27 Brandtzaeg P, Farstad IN, Johansen FE, et al. The B cell system of human mucosae and exocrine glands. Immunol Rev 1999;171:45-87.

28 Brandtzaeg $\mathbf{P}$. Distribution and characteristics of mucosal immunoglobulin-producing cell. In: Ogra PL, ed. Handbook of mucosa Immunology. San Diego: Academic Press, 1994:251-62.

29 Macpherson AJ, Gatto D, Sainsbury E, et al. A primitive T cell-independent mechanism of intestinal mucosal $\lg A$ response to commensal bacteria. Science 2000;288:2222-6

30 Fagarasan S, Kinoshita K, Maramatsu M, et al. In situ class-switching and differentiation to $\lg \mathrm{A}$-producing cells in the gut lamina propria. Nature 2001:413:639-43.

31 Fischer M, Kuppers R. Human IgA- and IgM-secreting intestinal plasma cells carry heavily mutated $\mathrm{VH}$ region genes. Eur J Immunol 1998;28:2971-77

32 Turley $\mathbf{H}$, Jones $M$, Erber $W$, et al. VS38: a new monoclonal antibody for detecting plasma cell differentiation in routine sections. J Clin Pathol 1994:47:418-22

33 Korsmeyer S. Bcl-2: a repressor of lymphocyte death. Immunol Today $1992 ; 13: 285-8$

34 Nutt S, Heavey B, Rollink A, et al. Commitment to the B-lymphoid lineage depends on the transcription factor pax5. Nature 1999:401:556-62

35 Turner CA, Mack D, Davis M. Blimp-1, a novel zinc finger-containing protein that can drive the maturation of $B$ lymphocytes into immunoglubulin-secreting cells. Cell 1994;77:297-306.

36 Huang S. Blimp- 1 is the murine homolog of the human transcriptional repressor PRDI-BF1. Cell 1994;78:9.

37 Angelin-Duclos C, Cattoretti G, Lin KL, et al. Commitment of B lymphocytes to a plasma cell fate is associated with blimp-1 expression in vivo. J Immunol 2000:165:5462-71.

38 Farstad IN, Halstensen TS, Lazarovits Al, et al. Human Intestinal B-cell blasts and plasma cells express the mucosal homing receptor integrin $\alpha 4 \beta 7$. Scand J Immunol 1995;42:662-72.

39 Siegel RM, Frederiksen JK, Zacharias DA, et al. Fas preassociation required for apoptosis signalling and dominant inhibition by pathogenic mutations. Science 2000:288:2354-7.

40 Neurath $M$, Stüber E, Strober W. BSAP: a key regulator of B-cell development and differentiation. Immunol Today 1995; 16:564-9.

41 Barberis A, Widenhorn K, Vitelli L, et al. A novel B-cell-lineage-specific transcription factor present at early but not late stages of differentiation. Gene Dev 1990;4:849-59

42 Usui T, Wakatsuki $Y$, Matsunaga, $Y$, et al. Overexpression of $B$ cell-specific activator protein (BSAP/Pax-5) in a late B cell is sufficient to suppress differentiation to an lg high producer cell with plasma cell suppress differentiation to an lg high producer
phenotype. J Immunol 1997;158:3197-204.

43 Berlin C, Bargatze RF Campbell JJ, et al. $\alpha 4$ integrin mediates lymphocyte attachment and rolling under physiologic flow. Cell 1995;80:413-22.

44 Bargatze RF, Jutila MA, Butcher EC. Distinct role of L-selectin and integrins $\alpha 4 \beta 7$ and LFA- 1 in lymphocytes homing to Peyer's patch-HEV in situ: the multistep model confirmed and refined. Immunity 1995:3:99-108

45 Wagner $\mathbf{N}$, Lohler J, Kunkel EJ, et al. Critical role for $\beta 7$ integrins in formation of the gut-associated lymphoid tissue. Nature 1996:382:366-70.

46 Salmi M, Andrew DP, Butcher EC, et al. Dual binding capacity of mucosal immunoblasts to mucosal and synovial endothelium in humans: Dissection of the molecular mechanisms. J Exp Med 1995;181:137-49.

47 Farsatad IN, Norsten J, Brandtzaeg P. Phenotypes of B and T cells in human intestinal and mesenteric lymph. Gastroenterology 1997; 112:163-73.

48 Kantele A, Kantele JM, Savilahti E, et al. Homing potentials of circulating lymphocytes in humans depend on the site of activation. Oral but not parenteral, typhoid vaccination induces circulating antibody-secreting cells that all bear Homing receptors directing them to the gut. J Immunol 1997; 158:574-57. 